\title{
New families of bivariate copulas via unit weibull distortion
}

\author{
Fadal A.A. Aldhufairi* (D) and Jungsywan H. Sepanski
}

\author{
*Correspondence: \\ aldhu1fa@cmich.edu \\ Department of Statistics, Actuarial \\ and Data Sciences, Central \\ Michigan University, Mount \\ Pleasant, MI 48859 USA
}

\begin{abstract}
This paper introduces a new family of bivariate copulas constructed using a unit Weibull distortion. Existing copulas play the role of the base or initial copulas that are transformed or distorted into a new family of copulas with additional parameters, allowing more flexibility and better fit to data. We present a general form for the new bivariate copula function and its conditional and density distributions. The tail behaviors are investigated and indicate the unit Weibull distortion may result in new copulas with upper tail dependence when the base copula has no upper tail dependence. The concordance ordering and Kendall's tau are derived for the cases when the base copulas are Archimedean, such as the Clayton and Frank copulas. The Loss-ALEA data are analyzed to evaluate the performance of the proposed new families of copulas.
\end{abstract}

Keywords: Archimedean copula, Distortion function, Kendall's tau, Weibull distribution, Tail dependence coefficient, Tail order

\section{Introduction}

Copulas serve numerous fields including insurance and finance. For example, (Frees and Valdez 1998) demonstrated their usefulness and explored practical applications such as estimation of joint life mortality and multi-decrement models. Nazemi and Elshorbagy (2012) implemented copula modeling to study the interdependence among hydrological data. The fitness of statistical models rests on its flexibility and more parameters may better accommodate various features in data. Construction of new families of copulas with better fitness has been of interest to researchers. In this paper, we provide a new distortion mechanism of copula construction and start by setting forth fundamentals and relevant literature below.

Let $X$ and $Y$ be continuous random variables with a joint distribution function $H(x, y)=$ $P(X \leq x, Y \leq y)$ and marginal cumulative distribution functions (cdf) $F(x)=P(X \leq x)$ and $G(y)=P(Y \leq y)$. Sklar (1959) showed that there exists a unique copula $C$ such that $H(x, y)=C(F(x), G(y))$ with joint probability density function (pdf), $h(x, y)$, given by

$$
h(x, y)=c(F(x), G(y)) f(x) g(y)
$$

where the copula pdf $c(u, v)=\partial C(u, v) / \partial u \partial v, f(x)=d F(x) / d x=F^{\prime}(x)$, and $g(y)=$ $d G(y) / d y=G^{\prime}(y)$. Note the prime mark will be used to denote a derivative throughout the paper.

(c) The Author(s). 2020 Open Access This article is licensed under a Creative Commons Attribution 4.0 International License, which permits use, sharing, adaptation, distribution and reproduction in any medium or format, as long as you give appropriate credit to the original author(s) and the source, provide a link to the Creative Commons licence, and indicate if changes were made. The images or other third party material in this article are included in the article's Creative Commons licence, unless indicated otherwise in a credit line to the material. If material is not included in the article's Creative Commons licence and your intended use is not permitted by statutory regulation or exceeds the permitted use, you will need to obtain permission directly from the copyright holder. To view a copy of this licence, visit http://creativecommons.org/licenses/by/4.0/. 
A bivariate copula can arise form a bivariate joint cdf. For example, the Gaussian copula is derived from the bivariate Gaussian distribution. Conversely, it can also be used to generate new bivariate probability distributions via (1); see Nelsen (2006) for summaries of methods of constructing copulas. Methods for constructing new bivariate joint distributions (Balakrishnan and Lai 2009) have also been adopted to create new copulas. For example, the framework of the Sarmanov-Lee distribution (Lee 1996) was utilized by Sharifonnasabi et al. (2018) and Cooray (2019) to construct new copulas. It is related to the bivariate FGM distribution introduced by Morgenstern (1956), given by $h(x, y)=$ $f(x) g(y)\left\{1+\alpha h_{1}(x) h_{2}(y)\right\}, x, y \in \mathcal{R}$, where $h_{1}$ and $h_{2}$ are two functions satisfying certain conditions.

Distortion or transformation of existing copulas is another framework for forging new families of copulas. Valdez and Xiao (2011)proposed three kinds of distortion approaches: (1) distortion of the margins alone without altering the original copula structure; (2) simultaneous distortion of the margins and the copula structure; and (3) synchronized distortion of the copula and its margins. In this paper, we focus on the distortion of the third kind that acts on the copula and induces the copula defined in (2). A function $T:[0,1] \rightarrow[0,1]$ is said to be a distortion function if it is continuous and non-decreasing, not necessarily convex or concave, with $T(0)=0$ and $T(1)=1$. It is said to be admissible for a base or an initial copula $C$ if the transformed copula $C_{T}(u, v)$ of the form

$$
C_{T}(u, v)=T\left(C\left(T^{-1}(u), T^{-1}(v)\right)\right), \text { for } u, v \in I,
$$

is also a copula. Note that, as in Valdez and Xiao (2011), $T$ is assumed to be strictly increasing such that $T^{-1}$ exists and is continuous on $[0,1]$.

If the initial copula is Archimedean with generator $\phi$, then $C_{T}$ is Archimedean with generator $\phi \circ T^{-1}$; see Di Bernardino and Rulliere (2013) and the right composition rule in Genest et al. (1998). A convex $T$ is required for the admissibility; see Morillas (2005) or Theorem 3.3.3 in Nelsen (2006). Durante et al. (2010) showed $T$ is admissible if $T$ 。 $\exp :(-\infty, 0) \rightarrow[0,1]$ is log-convex and suggested several distortion functions. The logconvex condition will be used to obtain the admissible parameter space for the proposed distortion. Samanthi and Sepanski (2019) constructed a new family of copulas via beta cdf distortion. The mixture of Max-infinitely divisible approach for constructing BB1-BB7 copulas in Joe (2015) is also a distortion method. Xie et al. (2019) presented a family of bivariate copulas by transforming an initial/base copula using two increasing functions. For more references, see Xie et al. (2019).

In this paper, we inaugurate a distributional distortion derived by a transformation of a Weibull random variable. This paper is organized as follows. In "Groundwork", we first lay some groundwork required for the derivation of properties of the new family of copulas. "The proposed unit weibull distortion" stages the unit Weibull (UW) distortion function and the admissibility conditions on the parameters. In "Unit-Weibull distorted copulas", the UW distorted copula distribution and its corresponding conditional and density distributions are formulated. Examples and possible limiting cases in parameters are presented. "Properties" investigates properties such as the tail dependence coefficients, tail orders, and concordance ordering. To assess its performance, the new UW-distorted copula model is applied to the Loss-ALAE data set in "Application" sections, followed by concluding remarks. 


\section{Groundwork}

In this section, we describe notation, definitions and some known results that would be applied to distorted copulas; see Joe (2015) for more details.

From (1), a copula contains the dependence structure between two random variables and links a bivariate distribution function to its univariate marginal cdf's. It has the following properties: i) $C(u, 0)=C(0, v)=0,(u, v) \in I^{2}$, where $I=[0,1]$; ii) $C(u, 1)=u$ and $C(1, v)=v,(u, v) \in I^{2}$; and iii) $C\left(u_{2}, v_{2}\right)-C\left(u_{2}, v_{1}\right)-C\left(u_{1}, v_{2}\right)+C\left(u_{1}, v_{1}\right) \geq 0$, for $u_{1} \leq u_{2}, v_{1} \leq v_{2}$, and $\left(u_{1}, u_{2}\right),\left(v_{1}, v_{2}\right) \in I^{2}$. A copula $C$ is Archimedean with strict generator $\phi$ if it admits the representation of $\phi^{-1}(\phi(u)+\phi(v))$, where $\phi: I \rightarrow[0, \infty]$ is a continuous, strictly decreasing and convex function such that $\phi(1)=0$ and $\phi(0)=\infty$.

Tail dependence coefficients are measures of extremal dependence that quantify the dependence in the lower-left-quadrant tail or upper-right-quadrant tail of a bivariate distribution. Let $U$ and $V$ be two unit uniform random variables with a joint copula cdf $C(u, v)=\operatorname{Pr}(U \leq u, V \leq v), u, v \in I$. The lower tail dependence coefficient, $\lambda_{L}$, is defined as the limit value of the conditional probability of $U \leq u$ given $V \leq u$ as $u \rightarrow 0^{+}$and can be calculated as $\lim _{u \rightarrow 0^{+}} C(u, u) / u$. The upper tail dependence coefficient, $\lambda_{U}$, is defined as the limit value of the conditional probability of $U>u$ given $V>u$ as $u \rightarrow 1^{-}$. It can be simplified as $\lim _{u \rightarrow 1^{-}} \bar{C}(u, u) /(1-u)$, where $\bar{C}(u, v)=P(U>u, V>v)$.

Let $T$ be an admissible distortion function, then the induced copula is of the form displayed in (2). Since $T$ is a distortion function and by L'Hopital's rule, the lower tail dependence coefficient for a T-distortion induced copula is given by

$$
\begin{aligned}
\lambda_{T, L} & =\lim _{u \rightarrow 0^{+}} \frac{T\left(C\left(T^{-1}(u), T^{-1}(u)\right)\right)}{u} \\
& =\lim _{u \rightarrow 0^{+}} \frac{T(C(u, u))}{T(u)}=\lim _{u \rightarrow 0^{+}} \frac{t(C(u, u))}{t(u)} \frac{d C(u, u)}{d u},
\end{aligned}
$$

where $t(u)=d T(u) / d u$, if the lower tail dependence coefficient $\lambda_{L}$ of the initial copula $C$ and the limit of $t(C(u, u)) / t(u)$ at $0^{+}$exist. Since $\lim _{u \rightarrow 1^{-}} T(u)=1$, with the substitution of $v=T^{-1}(u)$ and by L'Hopital's rule, the upper tail dependence coefficient is given by

$$
\begin{aligned}
\lambda_{T, U} & =2-\lim _{u \rightarrow 1^{-}} \frac{1-T\left(C\left(T^{-1}(u), T^{-1}(u)\right)\right)}{1-u} \\
& =2-\lim _{v \rightarrow 1^{-}} \frac{1-T(C(v, v))}{1-T(v)}=2-\lim _{v \rightarrow 1^{-}} \frac{t(C(v, v))}{t(v)} \frac{d C(v, v)}{d v}
\end{aligned}
$$

if the upper tail dependence coefficient $\lambda_{U}$ of the initial copula $C$ and the limit of $t(C(u, u)) / t(u)$ at $1^{-}$exit.

Let $f_{1}$ and $f_{2}$ be two functions. If $\lim _{u \rightarrow u_{0}} f_{1}(u) / f_{2}(u)=1$, we denote it by $f_{1}(u) \sim f_{2}(u)$ as $u \rightarrow u_{0}$. A positive function $f_{1}$ defined on $(0, \infty)$ is regularly varying at 0 with index $\gamma$, in which case we write $f_{1} \in \mathcal{R}(\gamma)$, if for some real number $\gamma$ it satisfies

$$
\lim _{x \rightarrow 0} f_{1}(s x) / f_{1}(x)=s^{\gamma} \text { for all } s>0 .
$$

A function $f_{1}$ is said to be slowly varying if $\gamma=0$. Karamata's Characterization Theorem (Bingham et al. 1989) states that every regularly varying function $f_{1}$ with index $\gamma$ is of the form $f_{1}(x)=x^{\gamma} \ell(x)$, where $\ell$ is a slowly varying function. Buldygin et al. (2006) derived that if $f_{1}(x)$ is regularly varying at 0 (or $\infty$ ) with an order of $\gamma \in \mathcal{R}$, then

$$
\lim _{x \rightarrow 0^{+}} \frac{\log f_{1}(x)}{\log x}=\gamma
$$


For a bivariate copula $C$, if $C(u, u) \sim u^{\kappa_{L}} \ell(u)$ as $u \rightarrow 0^{+}$, where $\ell(u)$ is slowly varying at $0^{+}$, then $\kappa_{L}$ is referred to as the lower tail order of the copula $C$. Let $\widehat{C}(u, v)=\bar{C}(1-u, 1-$ $v)=u+v-1+C(1-u, 1-v)$ be the survival copula. The upper tail order is defined as $\kappa_{U}$ if $\widehat{C}(u, u) \sim u^{\kappa} \ell_{*}(u)$ as $u \rightarrow 0^{+}$for some slowly varying function $\ell_{*}(u)$. When $\kappa_{L}=2$ and $\ell(u) \rightarrow q$ as $u \rightarrow 0^{+}$, for some positive $q$, the variables are near independent in the lower tail. If $1<\kappa_{L}<2$, the variables are positively associated and have intermediate tail dependence. The case $\kappa_{L}=1$ corresponds to the usual tail dependence coefficient $\lambda_{L} \in(0,1)$ with $\lim _{u \rightarrow 0^{+}} C(u, u) / u=\lim _{u \rightarrow 0^{+}} \ell(u)$. Similar conclusions are made for the upper tail and $\kappa_{U}$; see Hua and Joe (2013) for more details.

\section{The proposed unit weibull distortion}

By the definition of a distortion function, a continuous cdf with domain $I$ is a distortion function. In this section, we define the unit Weibull cdf and examine its admissibility.

Let $W$ be a non-negative continuous random variable with $\operatorname{cdf} G($.$) and \operatorname{pdf} g(\cdot)$. Define $Z=\exp (-W)$. Then, the $\operatorname{cdf}$ of $Z$ is given by

$$
T(z)=P(Z \leq z)=P(W \geq-\log z)=1-G(-\log z) \text { for } z \in I,
$$

which is the survival function of $W$ evaluated at $-\log z$. It is related to the expression $\bar{G}(-\log z)$, where $\bar{G}$ is a survival function, suggested by Durante et al. (2010). If $W$ is a Weibull random variable, we name $Z$ as a unit Weibull (UW) random variable with a support of the unit interval $I$. Let $G$ be the Weibull cdf given by $G(w)=$ $1-\exp \left(-b w^{a}\right), a, b>0, w \geq 0$, then the $\operatorname{cdf}$ of $Z$ is given by

$$
T(z)=\exp \left(-b(-\log z)^{a}\right) .
$$

The UW quantile function and UW pdf are given by, respectively,

$$
\begin{aligned}
T^{-1}(z) & =\exp \left[-\left(-\frac{1}{b} \log z\right)^{1 / a}\right], \\
t(z) & =\frac{a b}{z}(-\log z)^{a-1} \exp \left(-b(-\log z)^{a}\right) .
\end{aligned}
$$

To find the admissibility of the distributional distortion function $T$, we employ the following proposition shown in Durante et al. (2010).

Proposition 1 Let $T$ be an increasing bijective distortion. If $T \circ \exp :(-\infty, 0] \rightarrow[0,1]$ is log-convex, then the function $C_{T}$ in (2) is a copula.

The following corollary specifies constraints on the parameter values in the UW distortion to ensure the admissibility.

Corollary 1 Let $T(u)$ and $T^{-1}(u)$ for $u \in[0,1]$ be the $U W$-distortion and quantile functions in (7) and (8), respectively. Then, the function $C_{T}$ in (2) is a copula if $0<a \leq 1$ and $b>0$.

Proof Note $T \circ$ exp is log-convex if $\log \circ T \circ$ exp is convex. Define $l(x)=$ $\log (T[\exp (x)])=-b(-x)^{a}, x \in(-\infty, 0]$. The first and second derivatives of $l(\cdot)$ are

$$
l^{\prime}(x)=a b(-x)^{a-1} \text { and } l^{\prime \prime}(x)=a b(1-a)(-x)^{a-2},
$$


respectively. Since the second derivative $l^{\prime \prime}$ is non-negative if $0<a \leq 1$ and $b>0$, the conclusion follows from Proposition 1.

\section{Unit-Weibull distorted copulas}

We below present the copula distribution, pdf, and conditional pdf, and derive limiting cases for the proposed new family of UW distorted copulas.

Applying (7) and (8), the copula $C_{T}$ in (2) is of the following general form

$$
C_{T}(u, v)=\exp \left\{-b\left[-\log C\left(e^{-\left(-b^{-1} \log u\right)^{1 / a}}, e^{-\left(-b^{-1} \log v\right)^{1 / a}}\right)\right]^{a}\right\}
$$

where $0<a \leq 1$ and $b>0$. When $a=1$ and $b=1$, then $T(u)=u$, i.e., the initial copula is not distorted. The initial copula is a member of the proposed family of copulas. When $a=1$, the UW distortion is the power distortion.

If the initial copula $C$ is Archimedean with a strict generator function of $\phi$, then $C_{T}$ is Archimedean with generator given by

$$
\Phi(u)=\phi\left(T^{-1}(u)\right)=\phi\left\{\exp \left[-\left(-\frac{\log u}{b}\right)^{1 / a}\right]\right\}, u \in I .
$$

Example 1 UW-Clayton copula. Consider the Clayton copula expressed as $C(u, v ; \theta)=$ $\left(u^{-\theta}+v^{-\theta}-1\right)^{-1 / \theta}, \theta>0$ with generator given by $\left(t^{-\theta}-1\right) / \theta$. The UW-Clayton copula has the following expression

$$
\begin{aligned}
C_{T}(u, v) & =T\left\{\left[\left(T^{-1}(u)\right)^{-\theta}+\left(T^{-1}(v)\right)^{-\theta}-1\right]^{-1 / \theta}\right\} \\
& =\exp \left\{-b\left[\frac{1}{\theta} \log \left(\exp \left[\theta\left(-\frac{1}{b} \log u\right)^{1 / a}\right]+\exp \left[\theta\left(-\frac{1}{b} \log v\right)^{1 / a}\right]-1\right)\right]^{a}\right\},
\end{aligned}
$$

and its generator is given by $\Phi(u)=\left\{\exp \left[\theta\left(-b^{-1} \log u\right)^{1 / a}\right]-1\right\} / \theta$. The bivariate BB3 copula derived by Joe (2015) is a special case when $b=1$.

Example 2 UW-Gumbel copula. Consider the Gumbel copula expressed as $C(u, v ; \theta)=$ $\exp \left\{-\left[(-\log u)^{\theta}+(-\log v)^{\theta}\right]^{1 / \theta}\right\}, \theta \geq 1$. It is Archimedean with generator $(-\log t)^{\theta}$. The UW-Gumbel copula has the following expression

$$
C_{T}(u, v)=\exp \left\{-\left[(-\log u)^{\theta / a}+(-\log v)^{\theta / a}\right]^{a / \theta}\right\} .
$$

Note the parameters $\theta$ and $a$ cannot be identified separately. Reparameterizing by setting $\theta / a=\delta$, we see that the UW distortion of the Gumbel copula returns the Gumbel copula and does not yield a new family of copulas.

This example prompts us to consider the UW distortion of extreme-value bivariate copulas such that $C\left(u^{1 / m}, v^{1 / m}\right)^{m}=C(u, v)$, for integers $m \geq 1$, and are of the form

$$
C(u, v)=\exp \left[\log (u v) A\left(\frac{\log (v)}{\log (u v)}\right)\right], u, v \in(0,1)
$$

where $A(\cdot)$ is convex and satisfies certain constraints; see Gudendorf and Segers (2010). In this case, since $T^{-1}(u)=\exp \left[-\left(-b^{-1} \log u\right)^{1 / a}\right]$,

$C_{T}(u, v)=\exp \left[-\left\{-\left[(-\log u)^{1 / a}+(-\log v)^{1 / a}\right] A\left(\frac{(-\log v)^{1 / a}}{(-\log u)^{1 / a}+(-\log v)^{1 / a}}\right)\right\}^{a}\right]$. 
The parameter $b$ originated from the UW distortion disappears.

Example 3 UW-independence copula. Consider the independence copula expressed as $C(u, v)=u v$. The UW-independence copula has the following expression

$$
\begin{aligned}
C_{T}(u, v) & =\exp \left\{-b\left[\left(-\frac{1}{b} \log u\right)^{1 / a}+\left(-\frac{1}{b} \log v\right)^{1 / a}\right]^{a}\right\} \\
& =\exp \left\{-\left[(-\log u)^{1 / a}+(-\log v)^{1 / a}\right]^{a}\right\} .
\end{aligned}
$$

Therefore distorting the independence copula results in the Gumbel copula. That is, the proposed UW distortion gives another genesis of the Gumbel copula.

Example 4 UW-Frank copula. The Frank copula is defined as $C(u, v ; \theta)=$ $-\theta^{-1} \log \left\{1+\left[\left(e^{-\theta u}-1\right)\left(e^{-\theta v}-1\right)\right] /\left(e^{-\theta}-1\right)\right\}, \theta \neq 0$, with generator function $-\log \left[\left(e^{-\theta t}-1\right) /\left(e^{-\theta}-1\right)\right]$. The UW-Frank copula has the following expression

$$
C_{T}(u, v)=\exp \left\{-b\left[-\log \left(-\frac{1}{\theta} \log \left(1+\frac{[B(u)-1][B(v)-1]}{e^{-\theta}-1}\right)\right)\right]^{a}\right\},
$$

where $B(s)=\exp \left(T^{-1}(u)\right)=\exp \left(-\theta e^{-\left(-b^{-1} \log s\right)^{1 / a}}\right)$. Its generator is defined as $\Phi(u)=-\log \left\{[B(u)-1] /\left(e^{-\theta}-1\right)\right\}$.

\section{Conditional distribution and copula density}

The conditional density $C(u \mid v)$ plays a key role in simulating bivariate data linked by a copula $C$ since the conditional distribution $P(X \leq x \mid Y=y)=\partial C(F(x), G(y)) / \partial v$ and $C(u \mid v)=\partial C(u, v) / \partial v$. A general algorithm to generate draws from a bivariate copula $C$ using the conditional distribution approach (see Nelsen (2006)) is described as follows. (i) Generate two independent uniform random values $\left(u_{1}, v\right)$ and (ii) solve $C\left(u_{2} \mid u_{1}\right)-v=0$ for $u_{2}$. The desired pair is $\left(u_{1}, u_{2}\right)$. We will also obtain the copula density function needed for computing the maximum likelihood estimates of parameters.

Let $x=\exp \left\{-\left[-b^{-1} \log u\right]^{1 / a}\right\}$ and $y=\exp \left\{-\left[-b^{-1} \log v\right]^{1 / a}\right\}$; both are monotonically increasing. The inverse transforms are $u=\exp \left[-b(-\log x)^{a}\right]$ and $v=$ $\exp \left[-b(-\log y)^{a}\right]$. The copula $C_{T}$ in (10) can be rewritten as

$$
H(x, y)=C_{T}(u, v)=\exp \left\{-b[-\log C(x, y)]^{a}\right\} .
$$

The conditional cdf and copula pdf can be respectively derived by

$$
C_{T}(v \mid u)=\frac{\partial H}{\partial x} \frac{\partial x}{\partial u} \text { and } c_{T}(u, v)=\frac{\partial^{2} H}{\partial y \partial x} \frac{\partial x}{\partial u} \frac{\partial y}{\partial v} .
$$

The derivatives of $H$ with respect to $x$ and $x$ with respect to $u$ are

$$
\begin{aligned}
& \frac{\partial H}{\partial x}=\frac{a b}{C(x, y)} \frac{\partial C(x, y)}{\partial x} H(x, y)[-\log C(x, y)]^{a-1}, \\
& \frac{\partial x}{\partial u}=\frac{x}{a b u}\left(-\frac{\log u}{b}\right)^{1 / a-1} .
\end{aligned}
$$

The conditional cdf is therefore given by

$$
C_{T}(v \mid u)=\frac{x H(x, y)}{b^{1 / a-1} C(x, y)} \frac{\partial C(x, y)}{\partial x}\left(\frac{(-\log u)^{1 / a}}{-\log C(x, y)}\right)^{1-a} \frac{1}{u} .
$$


If $a=b=1$, then $x=u, y=v$ and $C_{T}(v \mid u)=C(v \mid u)=\partial C(u, v) / \partial u$. The derivation of the copula density $c_{T}(u, v)$ in (12) requires the calculation of $\partial^{2} H / \partial y \partial x$, which is lengthy and tedious therefore not presented here.

Next, we consider the case when the initial copula is Archimedean with generator $\phi$. In this case, let $x=\phi\left[T^{-1}(u)\right]$ and $y=\phi\left[T^{-1}(v)\right]$. Note that $x$ and $y$ are decreasing and map $[0,1]$ to $[0, \infty]$ such that $\phi(1)=0$ and $\phi(0)=\infty$. Then,

$$
C_{T}(u, v)=e^{-b\left[-\log \phi^{-1}\left(\phi\left(T^{-1}(u)\right)+\phi\left(T^{-1}(v)\right)\right)\right]^{a}}=e^{-b\left[-\log \phi^{-1}(x+y)\right]^{a}}=\bar{H}(x, y),
$$

where $\bar{H}$ is a bivariate survival function with univariate margins $\exp \left\{-b\left[-\log \left(\phi^{-1}(x)\right)\right]^{a}\right\}$. Note that $d T^{-1}(u) / d u=1 / t\left(T^{-1}(u)\right)$, where $t(\cdot)$ is defined in (9). The conditional cdf and pdf of the UW distorted copula can be obtained from (12) and the following:

$$
\begin{aligned}
& C_{T}(v \mid u)=\frac{\partial \bar{H}}{\partial x} \frac{\partial x}{\partial u} ; \quad \frac{\partial x}{\partial u}=\frac{\partial}{\partial u}\left[\phi\left(T^{-1}(u)\right)\right]=\frac{\phi^{\prime}\left(T^{-1}(u)\right)}{t\left(T^{-1}(u)\right)} \\
& \frac{\partial \bar{H}(x, y)}{\partial x}=\frac{a b \bar{H}(x, y)}{\phi^{-1}(x+y)} \frac{\left[-\log \left(\phi^{-1}(x+y)\right)\right]^{a-1}}{\phi^{\prime}\left(\phi^{-1}(x+y)\right)}=\frac{a b \bar{H}(x, y) d_{1}^{a-1}}{d_{2}} \\
& \frac{\partial^{2} \bar{H}(x, y)}{\partial x \partial y}=\frac{a b \bar{H}(x, y) d_{1}^{a-1}}{d_{2}}\left\{\frac{a b d_{1}^{a-1}}{d_{2}}-\frac{(a-1)}{d_{1} d_{2}}-\frac{1}{d_{2}}-\frac{\phi^{\prime \prime}\left(\phi^{-1}(x+y)\right)}{\left[\phi^{\prime}\left(\phi^{-1}(x+y)\right)\right]^{2}}\right\}
\end{aligned}
$$

where $d_{1}=-\log \left(\phi^{-1}(x+y)\right)$ and $d_{2}=\phi^{-1}(x+y) \phi^{\prime}\left(\phi^{-1}(x+y)\right)$.

Example 5 UW-Clayton Copula. The Clayton copula, see Example 1, is Archimedean with generator $\phi(u)=\left(u^{-\theta}-1\right) / \theta$ and $\phi^{-1}(u)=(1+\theta u)^{-1 / \theta}$. Let $x=$ $\left\{\exp \left[\theta(-\log (u) / b)^{1 / a}\right]-1\right\} / \theta$ and $y=\left\{\exp \left[\theta(-\log (v) / b)^{1 / a}\right]-1\right\} / \theta$. One can plug in the following components into (13) and (14) to obtain the conditional distribution and density of the UW-Clayton copula:

$$
\begin{aligned}
& \phi^{-1}(x+y)=[1+\theta(x+y)]^{-1 / \theta} \\
& \phi^{\prime}\left(\phi^{-1}(x+y)\right)=-[1+\theta(x+y)]^{1+1 / \theta} ; \\
& \phi^{\prime \prime}\left(\phi^{-1}(x+y)\right)=(\theta+1)[1+\theta(x+y)]^{1+2 / \theta} .
\end{aligned}
$$

Example 6 UW-Frank Copula. The Frank copula is Archimedean with generator function $\phi(u)=-\log \left[\left(e^{-\theta u}-1\right) /\left(e^{-\theta}-1\right)\right]$ and $\phi^{-1}(u)=-\theta^{-1} \log \left(1+e^{-u}\left(e^{-\theta}-1\right)\right)$; see Example 4. In this case, $x=-\log \left\{\left[\exp \left(-\theta e^{-(-\log (u) / b)^{1 / a}}\right)-1\right] /\left(e^{-\theta}-1\right)\right\}$ and $y=-\log \left\{\left[\exp \left(-\theta e^{-(-\log (v) / b)^{1 / a}}\right)-1\right] /\left(e^{-\theta}-1\right)\right\}$. To use (13) and (14) to derive the conditional distribution and density of the UW-Frank copula, the following expressions will be required:

$$
\begin{aligned}
& \phi^{-1}(x+y)=-\theta^{-1} \log \left(1+e^{-(x+y)}\left(e^{-\theta}-1\right)\right) ; \\
& \phi^{\prime}\left(\phi^{-1}(x+y)\right)=\theta\left[1+e^{x+y}\left(e^{-\theta}-1\right)^{-1}\right] \\
& \phi^{\prime \prime}\left(\phi^{-1}(x+y)\right)=\theta^{2} e^{x+y}\left(e^{-\theta}-1\right)^{-1}\left[1+e^{x+y}\left(e^{-\theta}-1\right)^{-1}\right] .
\end{aligned}
$$




\section{Limiting cases}

When $a=1$, the UW distortion function becomes $T(u)=u^{b}$, the power distortion, and the UW distortion results in copulas of the form $C_{T}(u, v)=\left[C\left(u^{1 / b}, v^{1 / b}\right)\right]^{b}$. Proposition 2 below is not applicable to the case when the initial copula is an extreme-value copula, for the power distortion doesn't produce a new family of copulas in this case; see Example 2.

Proposition 2 Let $C_{T}$ be the unit-Weibull distorted copula in (10), where $0<a \leq 1$ and $b>0$. Then, $C_{T}$ approaches the independence copula when $b \rightarrow \infty$ and $a \rightarrow 1$.

Proof Let $r=1 / b, x=e^{-(-r \log u)^{1 / a}}, y=e^{-(-r \log v)^{1 / a}}$, and $A_{r}=C(x, y)$. The derivative $A_{r}^{\prime}=d A_{r} / d r$ is given by

$$
A_{r}^{\prime}=-\frac{r^{1 / a-1}}{a}\left\{C_{2 \mid 1}(x, y)\left[x(-\log u)^{1 / a}\right]+C_{1 \mid 2}(x, y)\left[y(-\log v)^{1 / a}\right]\right\},
$$

where $C_{2 \mid 1}(u, v)=\partial C(u, v) / \partial u$ and $C_{1 \mid 2}(u, v)=\partial C(u, v) / \partial v$. By L'Hopital's Rule and chain rule, the limit of the exponent term in (10) as $r \rightarrow 0$ or $b \rightarrow \infty$, is, if exists,

$$
\lim _{r \rightarrow 0} \frac{-\left[-\log A_{r}\right]^{a}}{r}=\frac{a\left[-\log A_{r}\right]^{a-1} A_{r}^{\prime}}{A_{r}}
$$

where $C_{2 \mid 1}(u, v)=\partial C(u, v) / \partial u$ and $C_{1 \mid 2}(u, v)=\partial C(u, v) / \partial v$. As $b \rightarrow \infty$ or $r \rightarrow 0$, we have that $x \rightarrow 1, y \rightarrow 1$, and $A_{r} \rightarrow 1$. When $a \rightarrow 1, \lim A_{r}^{\prime}=\log (u v)$ since $C_{2 \mid 1}$ and $C_{1 \mid 2}$ are conditional distributions. Therefore, $\lim _{b \rightarrow \infty} C_{T}(u, v)=\exp (\log (u v))=u v$.

Proposition 2 provides the limit of the UW copulas when $b \rightarrow \infty$ and $a \rightarrow 1$ without specifying the initial copula. In the following, we find the limiting copulas in the parameter $\theta$ originated from the initial copula for families of UW-Clayton and UW-Frank copulas.

Example 7 Consider UW-Clayton copula in Example 1. By the same arguments for the limit of the Clayton copula in Joe (2015),

$$
\begin{aligned}
& \lim _{\theta \rightarrow \infty} C\left(T^{-1}(u), T^{-1}(v)\right)=\min \left\{T^{-1}(u), T^{-1}(v)\right\}, \\
& \lim _{\theta \rightarrow 0^{+}} C\left(T^{-1}(u), T^{-1}(v)\right)=T^{-1}(u) T^{-1}(v) .
\end{aligned}
$$

Therefore, the UW-Clayton copula of the form $T\left(C\left(T^{-1}(u), T^{-1}(v)\right)\right)$, by Example 3,

$$
\begin{aligned}
& \lim _{\theta \rightarrow \infty} T\left(C\left(T^{-1}(u), T^{-1}(v)\right)\right)=\min \{u, v\}, \\
& \lim _{\theta \rightarrow 0^{+}} T\left(C\left(T^{-1}(u), T^{-1}(v)\right)\right)=\exp \left\{-\left[(-\log u)^{1 / a}+(-\log v)^{1 / a}\right]^{a}\right\}, 0<a \leq 1 .
\end{aligned}
$$

The limit of UW-Clayton copulas as $\theta \rightarrow \infty$ is the Gumbel copula.

When $b=1$, the UW-Clayton begets the BB3 copula. Therefore, UW-Clayton copulas approach the comonotonicity copula when $\theta \rightarrow \infty$ or $a \rightarrow 0^{+}$, and the Gumbel family when $\theta \rightarrow 0^{+}$. 
Example 8 Consider the UW-Frank copulas in Example 4. Let $x=T^{-1}(u)$ and $y=$ $T^{-1}(v)$. Following the arguments in Frank (1979), we have that

$$
\begin{aligned}
& -\frac{1}{\theta} \log \left(1+\frac{\left[e^{-\theta x}-1\right]\left[e^{-\theta y}-1\right]}{e^{-\theta}-1}\right)=x-\frac{1}{\theta} \log \left(\frac{e^{-\theta(1-x)}+e^{-\theta y}+e^{-\theta(y-x)}-1}{e^{-\theta}-1}\right), \\
& \Phi(u)=\phi(x)=-\log \left(\frac{e^{-\theta x}-1}{e^{-\theta}-1}\right) \rightarrow-\log x=-\log \left(T^{-1}(u)\right), \text { as } \theta \rightarrow 0^{+}, \\
& -\frac{1}{\theta} \Phi(u)=-\frac{1}{\theta} \phi(x)=1-x+\frac{1}{\theta} \log \left(\frac{1-e^{\theta x}}{1-e^{\theta}}\right) .
\end{aligned}
$$

For $0 \leq u \leq v \leq 1$ and $0 \leq x \leq y \leq 1$, as $\theta \rightarrow \infty$, the limit of (15) is $x=T^{-1}(u)$. Similarly, for $0 \leq v \leq u \leq 1$, as $\theta \rightarrow \infty$, the limit of (15) is $y=T^{-1}(v)$. Therefore, the limit of UW-Frank copula as $\theta \rightarrow \infty$ is the comonotonicity copula $\min (u, v)$.

As $\theta \rightarrow 0^{+}$, by (16) and the facts that $e^{-\theta} \sim 1-\theta,(1+s / n)^{n} \sim e^{s}$ as $n \rightarrow \infty$, using the Archimedean representation of the UW-Frank copula, the limit of the UW-Frank copula is

$$
T \circ \phi^{-1}(\phi(x)+\phi(y))=T \circ \phi^{-1}(\log (x y))=\exp \left[-\left((-\log u)^{1 / a}+(-\log v)^{1 / a}\right)^{a}\right],
$$

which is the Gumbel copula.

$$
\begin{aligned}
& \text { As } \theta \rightarrow-\infty \text {, by (17) and } \lim _{\theta \rightarrow-\infty} \phi(x)=0, \\
& \begin{aligned}
\phi^{-1}(\Phi(u)+\Phi(v)) & =-\frac{1}{\theta} \log \left(1-e^{-\phi(x)-\phi(y)}+\exp \left\{-\theta\left[1+\frac{\phi(x)}{\theta}+\frac{\phi(y)}{\theta}\right]\right\}\right) \\
& \rightarrow \max (x+y-1,0)=\max \left(T^{-1}(u)+T^{-1}(v)-1,0\right) .
\end{aligned}
\end{aligned}
$$

We therefore conclude that the limit of the UW-Frank copula as $\theta \rightarrow-\infty$ is

$$
\lim _{\theta \rightarrow-\infty} T \circ \phi^{-1}(\Phi(u)+\Phi(v))=\max \left[T\left(T^{-1}(u)+T^{-1}(v)-1\right), 0\right] .
$$

\section{Properties}

We obtain the tail dependence coefficients and tail orders for the UW-distorted copulas, and study the tail concordance in the parameters for the UW-Clayton copulas.

\section{Tail dependence coefficients and tail orders}

Consider the distortion $T(u)=\exp \left[-b(-\log u)^{a}\right]$, where $0<a \leq 1, b>0$, in (7). When $b=1, T$ turns into the form suggested by Durante et al. (2010), and when $b=1$ and $a=1, T$ is the identity distortion, i.e., no distortion is applied. Definitions of tail orders can be found in "Groundwork" section. We note here the joint survival function $\bar{C}(u, v)=P(U>u, V>v)=1-u-v+C(u, v)$ and Talyor's series approximation of

$$
(1+u)^{a} \sim 1+a u, \log (1-u) \sim-u, e^{u} \sim 1+u, \text { as } u \rightarrow 0 .
$$

Therefore, we have that

$$
T(1-u) \sim 1-b u^{a}, T^{-1}(1-u) \sim 1-(u / b)^{1 / a} \text { as } u \rightarrow 0^{+} .
$$

Below we assume that the lower tail coefficient $\lambda_{L}=0$ when $\kappa_{L}>1$ and the upper tail coefficient $\lambda_{U}=0$ when $\kappa_{U}>1$ for the initial copula. Let the subscript $T$ notation denote the properties of the UW distorted copulas, e.g., $\lambda_{T, U}$ is the lower tail coefficient for the UW distorted copulas. 
Proposition 3 Suppose that $C(u, u) \sim u^{\kappa_{L}} \ell(u)$ as $u \rightarrow 0^{+}$and $\bar{C}(1-u, 1-u) \sim$ $u^{\kappa} u \ell_{*}(u)$ as $u \rightarrow 0^{+}$for some slowly varying functions $\ell$ and $\ell_{*}$ at $0^{+}$. Then, for $C_{T}$ in (10), where $0<a \leq 1, b>0$,

(i) If $\kappa_{L}>1$, then $\kappa_{T, L}=\left(\kappa_{L}\right)^{a}$. If $\kappa_{L}=1$, then $\lambda_{T, L}=1$ for $a<1$ and $\lambda_{T, L}=\lambda_{L}^{b}$ for $a=1$.

(ii) $\kappa_{T, U}=1$ and $\lambda_{T, U}=2-\left(2-\lambda_{U}\right)^{a}$.

From (3), the lower tail dependence coefficient of $C_{T}$ is given by

$$
\begin{aligned}
\lambda_{T, L} & =\lim _{u \rightarrow 0^{+}} \frac{\exp \left[-b(-\log C(u, u))^{a}\right]}{\exp \left[-b(-\log u)^{a}\right]} \\
& =\lim _{u \rightarrow 0^{+}} \exp \left\{-b(-\log u)^{a}\left[\left(\frac{\log C(u, u)}{\log u}\right)^{a}-1\right]\right\} .
\end{aligned}
$$

By (5), $\lim _{u \rightarrow 0^{+}} \log C(u, u) / \log u=\kappa_{L}$. If $\kappa_{L}>1$, then $\lambda_{T, L}=0$ as $u \rightarrow 0^{+}$for $b>0$. If $\kappa_{L}=1$, applying L'Hopital's rule, the limit of the exponent in (20) is equal to

$$
\begin{aligned}
& -b \lim _{u \rightarrow 0^{+}}\left[\left(\frac{\log C(u, u)}{\log u}\right)^{a}-1\right] /(-\log u)^{-a} \\
& =b \lim _{u \rightarrow 0^{+}} u[-\log C(u, u)]^{a-1}(\log u)^{2} d\left[\frac{\log C(u, u)}{\log u}\right] / d u \\
& =b \lim _{u \rightarrow 0^{+}}[-\log C(u, u)]^{a-1}\left[\log C(u, u)-\frac{u}{C(u, u)} \frac{d C(u, u)}{d u} \log u\right] .
\end{aligned}
$$

If $\lim _{u \rightarrow 0^{+}} C(u, u) / u=\lim _{u \rightarrow 0^{+}} d C(u, u) / d u=\lambda_{L} \in(0,1], \lim _{u \rightarrow 0^{+}}[\log C(u, u)-$ $\log (u)]=\log \lambda_{L}$, then (21) is well defined. In this case, when $a<1$, the limit in (21) is 0 ; when $a=1$ it is equal to $b \log \lambda_{L}$. Thus, by (20) and (21), the lower tail dependence coefficient of the UW induced copula is given by

$$
\lambda_{T, L}=0 \text { if } \kappa_{L}>1 ; 1 \text { if } \kappa_{L}=1 \text { and } a<1 ; \lambda_{L}^{b} \text { if } \kappa_{L}=1 \text { and } a=1 .
$$

Proof By (4) and (9), the upper tail dependence coefficient is given by, if $\lambda_{U} \neq 0$,

$$
\begin{aligned}
\lambda_{T, U} & =2-\lim _{u \rightarrow 1^{-}} \frac{e^{-b([-\log C(u, u)])^{a}}}{e^{-b([-\log u])^{a}}}\left(\frac{\log C(u, u)}{\log u}\right)^{a-1} \frac{u}{C(u, u)} \frac{d C(u, u)}{d u} \\
& =2-\left(2-\lambda_{U}\right)^{a},
\end{aligned}
$$

by L'Hopital's rule and $\lim _{u \rightarrow 1^{-}} d C(u, u) / d u=2-\lambda_{U}$.

We obtain below tail orders of $C_{T}$. By (5) and (18) and since $T^{-1}(u)=$ $\exp \left[-\left(-b^{-1} \log u\right)^{1 / a}\right]$ and $\lim _{u \rightarrow 0^{+}} \log \ell(u) / \log u=0$ (Bingham et al. 1989),

$$
\begin{aligned}
T\left(C\left(T^{-1}(u), T^{-1}(u)\right)\right) & =\exp \left\{-b\left[-\log C\left(T^{-1}(u), T^{-1}(u)\right)\right]^{a}\right\} \\
& \sim \exp \left\{-b\left[-\kappa_{L}\left[\log T^{-1}(u)\right]\left(1+\frac{\log \ell\left(T^{-1}(u)\right)}{\kappa_{L} \log T^{-1}(u)}\right)\right]^{a}\right\} \\
& \sim \exp \left\{-b\left[-\kappa_{L} \log T^{-1}(u)\right]^{a}\left(1+a \frac{\log \ell\left(T^{-1}(u)\right)}{\kappa_{L} \log T^{-1}(u)}\right)\right\} \\
& \sim u^{\left(\kappa_{L}\right)^{a}} \exp \left\{-\left(\kappa_{L}\right)^{a-1} a b^{1 / a}(-\log u)^{1-1 / a} \log \ell\left(T^{-1}(u)\right)\right\} .
\end{aligned}
$$


Note that for $s>0$,

$$
\lim _{u \rightarrow 0^{+}} \frac{\ell\left(T^{-1}(s u)\right)}{\ell\left(T^{-1}(u)\right)}=\lim _{v \rightarrow 0^{+}} \frac{\ell\left(T^{-1}(s T(v))\right)}{\ell(v)}=\lim _{v \rightarrow 0^{+}} \frac{\ell(v q(v))}{\ell(v)}=1,
$$

where $T^{-1}(s T(v))=\exp \left\{-(-\log v)\left[1-(\log s) /\left(b(-\log v)^{a}\right)\right]^{1 / a}\right\}, \quad q(v)=$ $T^{-1}(s T(v)) / v$, and $\lim _{v \rightarrow 0^{+}} q(v)=1$. One can then show the exponential term in (22) is slowly varying by definition. Note that if $a=1$ and $b=1$, then (22) returns the assumption that $C(u, u) \sim u^{\kappa L} \ell(u)$ as $u \rightarrow 0^{+}$. Using the approximations in (18) and (19),

$$
\begin{aligned}
& {\left[-\log C\left(T^{-1}(1-u), T^{-1}(1-u)\right)\right]^{a} \sim\left[-\log C\left(1-\left(\frac{u}{b}\right)^{1 / a}, 1-\left(\frac{u}{b}\right)^{1 / a}\right)\right]^{a}} \\
& \sim\left[-\log \left(1-2\left(\frac{u}{b}\right)^{1 / a}+\bar{C}\left(1-\left(\frac{u}{b}\right)^{1 / a}, 1-\left(\frac{u}{b}\right)^{1 / a}\right)\right)\right]^{a} \\
& \sim \frac{2^{a} u}{b}\left[1-\frac{1}{2}\left(\frac{u}{b}\right)^{\left(\kappa_{U}-1\right) / a} \ell_{*}\left(\left(\frac{u}{b}\right)^{1 / a}\right)\right]^{a} \sim \frac{2^{a} u}{b}\left[1-\frac{a}{2}\left(\frac{u}{b}\right)^{\left(\kappa_{U}-1\right) / a} \ell_{*}\left(\left(\frac{u}{b}\right)^{1 / a}\right)\right] \\
& =E .
\end{aligned}
$$

Therefore, for the upper tail order, by (18),

$$
\begin{aligned}
\hat{C}_{T}(u, v) & \sim 2 u-1+\exp (-b E) \sim 2 u-b E \\
& \sim u\left\{2-2^{a}\left[1-\frac{a}{2}\left(\frac{u}{b}\right)^{\left(\kappa_{U}-1\right) / a} \ell_{*}\left(\left(\frac{u}{b}\right)^{1 / a}\right)\right]\right\},
\end{aligned}
$$

indicating that UW distorted copulas have an upper tail order of 1 except when $a=b=1$.

The following table summarizes tail orders and dependence coefficients for the family of UW-distorted copulas with the initial copulas being BB1, Clayton, Frank, and Gaussian copulas, where $\theta, \delta$, and $\rho$ are the parameters in the initial copulas with formulas shown in Joe (2015).

Density contour plots with standard normal margins for various combinations of $(a, b)$ are shown in Fig. 1. The parameter $\theta$ is chosen so that the initial copula has Kendall's tau of 2/7 or $-2 / 7$. As indicated by Proposition 3 or Table 1 , the family of UW-distorted copulas not only preserves the tail dependence of the initial copula but also can accommodate upper tail dependence. Unlike the initial Frank copula with $a=b=1$, the resulting UWFrank copulas are asymmetric. The graphs also reflect the results in Proposition 3, the upper tail dependence becomes stronger as $a$ decreases.

\section{Concordance ordering}

A copula is said to be positively ordered $C_{\alpha} \prec C_{\beta}$ if $C(u, v ; \alpha) \leq C(u, v ; \beta)$ whenever $\alpha \leq \beta$ for all $u, v \in I$, and negatively ordered $C_{\alpha} \succ C_{\beta}$ if $C(u, v ; \alpha)>C(u, v ; \beta)$ whenever the parameters $\alpha \leq \beta$ for all $u, v \in I$; see Nelsen (2006) for more details.

Proposition 4 If the initial copula is positively or negatively ordered by its parameter, then the unit-Weibull distortion preserves the concordance order in the parameter of the initial copula.

Proof If the initial copula $C$ is positively ordered, then, for $\theta_{1} \leq \theta_{2}$, $C\left(T^{-1}(u), T^{-1}(v) ; \theta_{1}\right) \leq C\left(T^{-1}(u), T^{-1}(v) ; \theta_{2}\right)$ for all $u, v \in I$. Let $T$ be the UW-distortion or an admissible distortion function. Since $T$ is increasing, with fixed $a$ and $b$ values, $T\left(C\left(T^{-1}(u), T^{-1}(v) ; \theta_{1}\right)\right) \leq T\left(C\left(T^{-1}(u), T^{-1}(v) ; \theta_{2}\right)\right)$, i.e., 


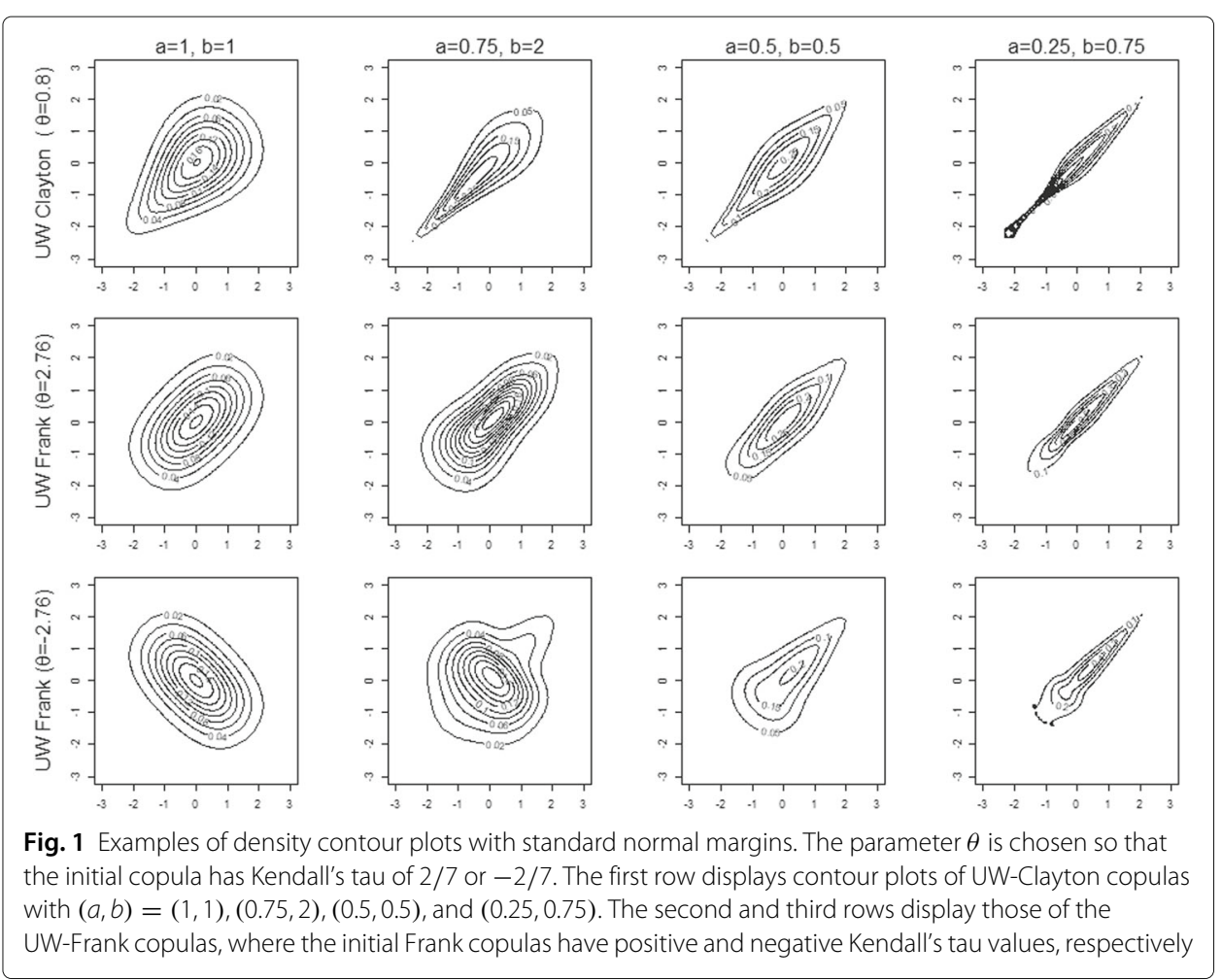

$C_{T}\left(u, v ; \theta_{1}\right) \leq C_{T}\left(u, v ; \theta_{2}\right)$. That is, the family of $T$-distortion induced copulas is also positively (negatively) ordered by the parameter $\theta$ originated from the initial copula if the initial copula is positively (negatively) ordered by the parameter.

To examine the concordance ordering in the parameters $a$ and $b$ introduced by the UW distortion for UW-Clayton copula, we present the following corollary and lemma; see Schweizer and Sklar (1983) or Nelsen (2006).

Corollary 2 Let $C_{1}$ and $C_{2}$ be Archimedean copulas with generators $\phi_{1}$ and $\phi_{2}$, respectively. Then $C_{1} \prec C_{2}$ holds if one of the following conditions is satisfied (i) $\phi_{1} \circ \phi_{2}^{-1}$

Table 1 Examples of tail orders and dependence coefficients

\begin{tabular}{lll}
\hline Initial Copula & $\kappa_{L}$ or $\lambda_{L}$ & $\kappa_{U}$ or $\lambda_{U}$ \\
\hline BB1 & $\lambda_{L}=2^{-1 / \theta \delta}$ & $\lambda_{U}=2-2^{1 / \delta}$ \\
Clayton & $\lambda_{L}=2^{-1 / \theta}$ & $\kappa_{U}=2$ \\
Frank & $\kappa_{L}=2$ & $\kappa_{U}=2$ \\
Gaussian & $\kappa_{L}=2 /(1+\rho)$ & $\kappa_{U}=2 /(1+\rho)$ \\
UW-Copula & $\kappa_{T, L}$ or $\lambda_{T, L}$ & $\kappa_{T, U}$ or $\lambda_{T, U}$ \\
UW-BB1 & $\lambda_{T, L}=2^{-b / \theta \delta}$ if $a=1$ & $\lambda_{T, U}=2-2^{a / \delta}$ \\
& $\lambda_{T, L}=1$ if $a<1$ & \\
UW-Clayton & $\lambda_{T, L}=2^{-b / \theta}$ if $a=1$ & $\lambda_{T, U}=2-2^{a}$ \\
& $\lambda_{T, L}=1$ if $a<1$ & \\
UW-Frank & $\kappa_{T, L}=2^{a}$ & $\lambda_{T, U}=2-2^{a}$ \\
UW-Gaussian & $\kappa_{T, L}=[2 /(1+\rho)]^{a}$ & $\lambda_{T, U}=2-2^{a}$ \\
\hline
\end{tabular}


is concave; (ii) $\phi_{1} / \phi_{2}$ is nondecreasing on (0,1); and (iii) $\phi_{1}$ and $\phi_{2}$ are continuously differentiable on $(0,1)$ and $\phi_{1}^{\prime} / \phi_{2}^{\prime}$ is nondecreasing on $(0,1)$.

Example 9 Consider the family of the UW-Clayton copulas in Example 1. Below we show the family of UW-Clayton copulas is negatively ordered by the parameter $b$ but not $a$. It is Archimedean, see Example 5, with generator and inverse generator given by

$$
\Phi(u ; a, b)=\frac{1}{\theta}\left[e^{\theta\left(-\frac{\log u}{b}\right)^{1 / a}}-1\right], \Phi^{-1}(u ; a, b)=e^{-b\left[\theta^{-1} \log (1+\theta u)\right]^{a}},
$$

respectively. We wish to use Corollary 2 to show the concordance order in the parameter and $b$. Define $h_{b}(u)$ to be

$h_{b}(u)=\Phi\left(u ; a, b_{1}\right) \circ \Phi^{-1}\left(u ; a, b_{2}\right)=\frac{1}{\theta}\left[e^{\left(b_{2} / b_{1}\right)^{1 / a} \log (1+\theta u)}-1\right]=\frac{1}{\theta}\left[(1+\theta u)^{\left(b_{2} / b_{1}\right)^{1 / a}}-1\right]$.

The first derivative and second derivatives of $h_{b}$ are give by

$$
\begin{aligned}
& h_{b}^{\prime}(u)=\left(b_{2} b_{1}^{-1}\right)^{1 / a}(1+\theta u)^{\left(b_{2} b_{1}^{-1}\right)^{1 / a}-1}, \\
& h_{b}^{\prime \prime}(u)=\theta\left(b_{2} b_{1}^{-1}\right)^{1 / a}\left[\left(b_{2} b_{1}^{-1}\right)^{1 / a}-1\right](1+\theta u)^{\left(b_{2} b_{1}^{-1}\right)^{1 / a}-2},
\end{aligned}
$$

which is negative if $b_{2}<b_{1}$ for $u \in(0,1]$. That is, the family of the UW-Clayton copulas is negatively ordered by the parameter $b$. Define $h_{a}(u)$ to be

$$
h_{a}(u)=\Phi\left(u ; a_{1}, b\right) \circ \Phi^{-1}\left(u ; a_{2}, b\right)=\frac{1}{\theta}\left[e^{\theta^{1-a_{2} / a_{1}}[\log (1+\theta u)]^{a_{2} / a_{1}}}-1\right] .
$$

The first derivative and second derivatives of $h_{a}$ are give by

$$
\begin{aligned}
& h_{a}^{\prime}(u)=\frac{a_{2} \theta^{1-a_{2} / a_{1}}}{a_{1}}\left[e^{\theta^{1-a_{2} / a_{1}}[\log (1+\theta u)]^{a_{2} / a_{1}}}[\log (1+\theta u)]^{a_{2} / a_{1}-1} \frac{1}{1+\theta u}\right], \\
& h_{a}^{\prime \prime}(u)=\frac{\theta h_{a}^{\prime}(u)}{1+\theta u}\left[\frac{a_{2} \theta^{1-a_{2} / a_{1}}}{a_{1}}[\log (1+\theta u)]^{a_{2} / a_{1}-1}+\frac{a_{2}-a_{1}}{a_{1} \log (1+\theta u)}-1\right] .
\end{aligned}
$$

The UW-Clayton is negatively ordered by the parameter $a$ if (23) is nonpositive for $a_{2}<a_{1}$ and all $u \in I$. As we will see from Fig. 2, the UW-Clayton is not ordered by the parameter $a$ for all $\theta$ and $b$ values.

\section{Measures of concordance}

In this section, we explore two widely known scale-invariant measures of concordance or association: Spearman's rho and Kendall's tau. If $X$ and $Y$ are continuous random variables with copula $C$, then the Spearman's rho and Kendall's tau can be expressed as, respectively,

$$
\begin{aligned}
& \rho_{S}=12 \int_{0}^{1} \int_{0}^{1} C(u, v) d u d v-3, \\
& \tau=1-4 \int_{0}^{1} \int_{0}^{1} \frac{\partial C}{\partial u}(u, v) \frac{\partial C}{\partial v}(u, v) d u d v .
\end{aligned}
$$

For an Archimedean copula (Genest and MacKay 1986), Kendall's tau is also given by

$$
\tau=1+4 \int_{0}^{1} \frac{\phi(u)}{\phi^{\prime}(u)} d u
$$




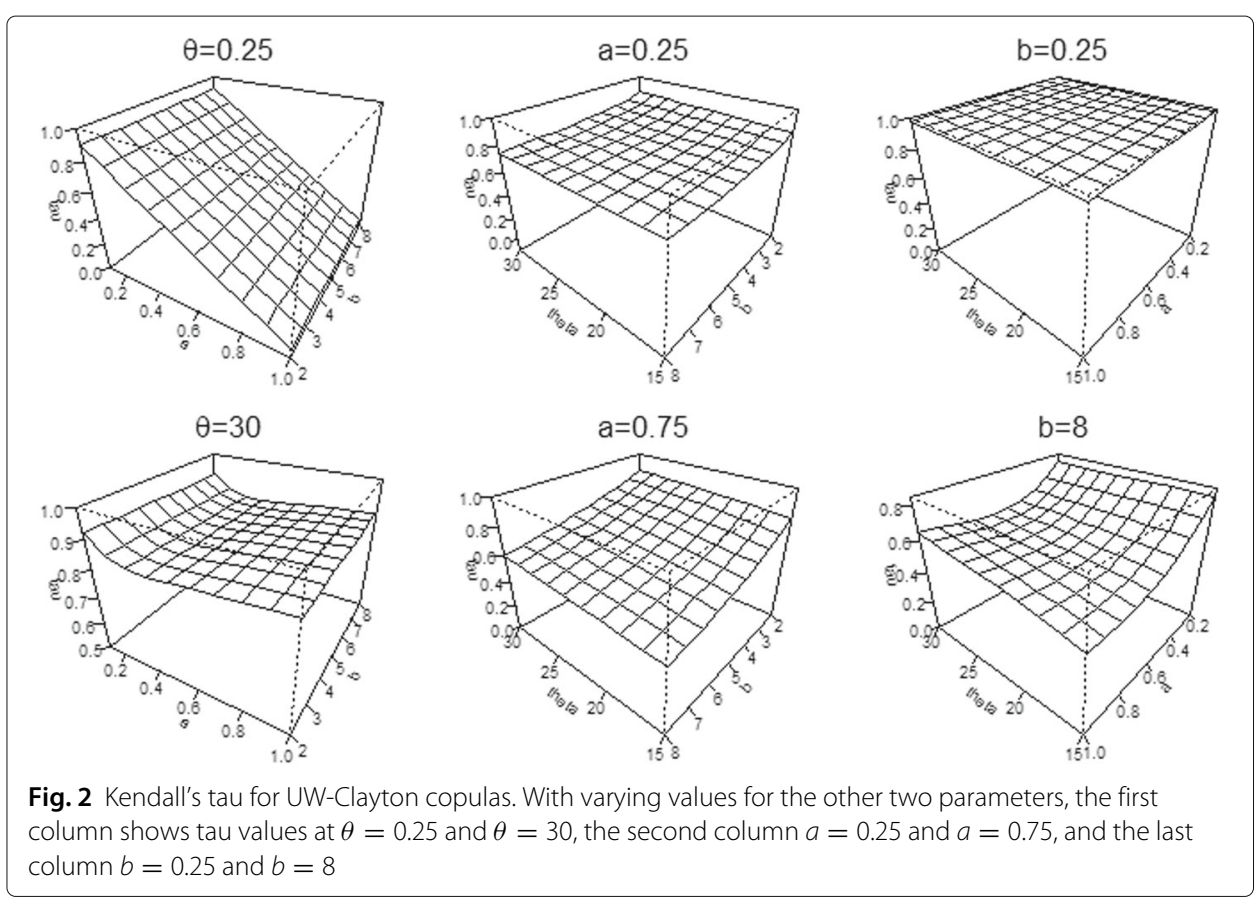

For the $T$-distortion induced copulas in (2), by substituting $T^{-1}(u)=x$ and $T^{-1}(v)=y$, Spearman's rho and Kendall's tau can be expressed as

$$
\begin{aligned}
& \rho_{T}=12 \int_{0}^{1} \int_{0}^{1} T(C(x, y)) t(x) t(y) d x d y-3 \\
& \tau_{T}=1-4 \int_{0}^{1} \int_{0}^{1}[t(C(x, y))]^{2} C_{1 \mid 2}(x \mid y) C_{2 \mid 1}(y \mid x) d x d y,
\end{aligned}
$$

where $t(v)=d T(v) / d v, C_{2 \mid 1}(u, v)=\partial C(u, v) / \partial u$ and $C_{1 \mid 2}(u, v)=\partial C(u, v) / \partial v$. Numerical integration methods can be employed to compute the concordance measures. If the inital copula is Archimedean with generator $\phi(u)$, from (11) and (24), $\Phi(u)=\phi\left(T^{-1}(u)\right)$ and $\Phi^{\prime}(u)=\phi^{\prime}\left(T^{-1}(u)\right) / t\left(T^{-1}(u)\right)$. The Kendall's tau for a UW distorted copula is given by

$$
\tau_{T}=1+4 \int_{0}^{1} \frac{\Phi(u)}{\Phi^{\prime}(u)} d u=1+4 \int_{0}^{1} \frac{\phi(v)}{\phi^{\prime}(v)} t^{2}(v) d v .
$$

Proposition 5 Let $X$ and $Y$ be random variables with copula $C_{T}$, a UW distorted copula of the form in (10), where the initial copula is Archimedean with generator $\phi(\cdot)$. Then, the Kendall's tau between $X$ and $Y$ can be expressed as

$$
1+4(a b)^{2} \int_{0}^{\infty} \frac{\phi\left(e^{-u}\right)}{\phi^{\prime}\left(e^{-u}\right)} u^{2 a-2} e^{u-2 b u^{a}} d u .
$$

Proof: From (9) and (25), with substitution $u=-\log v$, we have

$$
\tau_{T}=1+4(a b)^{2} \int_{0}^{\infty} \frac{\phi\left(e^{-u}\right)}{\phi^{\prime}\left(e^{-u}\right)} u^{2 a-2} e^{u-2 b u^{a}} d u .
$$


Example 10 Kendall's tau of the UW-Clayton copula. When the initial copula is the Clayton copula, Proposition 5 gives

$$
\begin{aligned}
& \tau_{T}=1-\frac{4(a b)^{2}}{\theta} \int_{0}^{\infty} u^{2 a-2}\left(1-e^{-\theta u}\right) e^{-2 b u^{a}} d u, \text { where } \\
& \int_{0}^{\infty} u^{2 a-2} e^{-2 u^{a}} d u=\frac{1}{a} \int_{0}^{\infty} v^{1-1 / a} e^{-2 v} d v=\frac{\Gamma(2-1 / a)}{a 2^{2-1 / a}} .
\end{aligned}
$$

With (26), one can readily write $\mathrm{R}$ programs to compute Kendall's tau values at various parameter values. Figure 2 plots values of Kendall's tau for various parameter values. The plot for $b=8$ indicates when $\theta$ is large, e.g., $\theta=30$, the family of UW-Clayton copulas is not ordered by the parameter $a$ as the resulting tau values are not monotone in $a$; see also Example 9.

Example 11 Kendall's tau of the UW-Frank copula. From Example 6 and Proposition 5, with substitution $v=\theta e^{-u}$, the Kendall's tau of a UW-Frank copula is given by

$$
\tau_{T}=1-4(a b)^{2} \int_{0}^{\theta} \frac{\left(1-e^{\nu}\right)[-\log (v / \theta)]^{2 a-2}}{v^{2} e^{2 b(-\log (v / \theta))^{a}}} \log \left(\frac{e^{-v}-1}{e^{-\theta}-1}\right) d v
$$

for $\theta \neq 0,0<a \leq 1$, and $b>0$. We compute Kendall's tau coefficients at various parameter values using the formula in (27) and produce Fig. 3. While not mathematically shown due to its tediousness, the plot for $\theta=10$ illustrates that the family of UW-Frank copulas is not ordered by the parameter $a$ as the resulting tau values are not monotone in a.

\section{Application}

In this section, the Loss-ALAE insurance data set is analyzed to evaluate the performance of the proposed UW distorted copula models. It is easily accessible from the R copula package. The loss variable is the general liability claims and the allocated loss adjustment

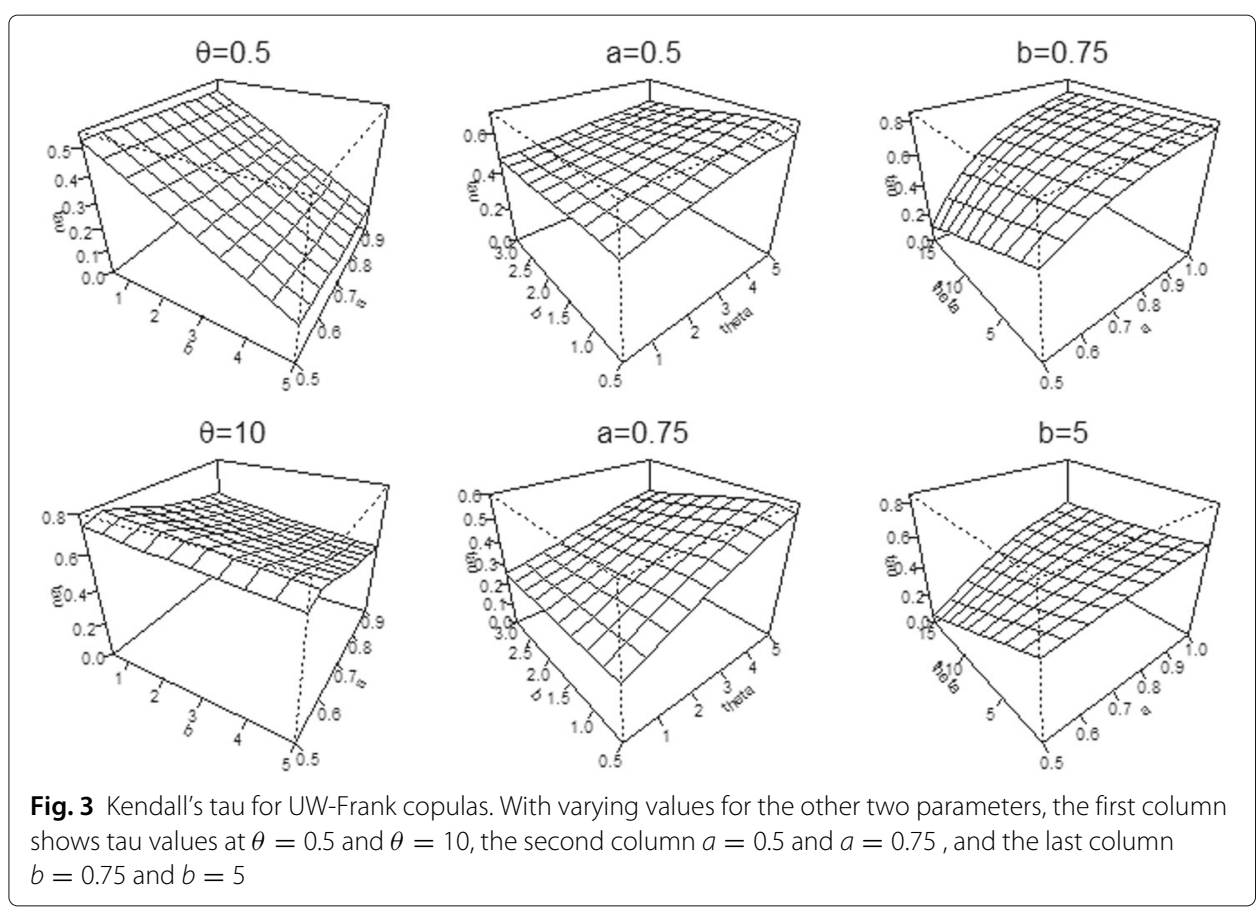


expenses (ALAE) is attributable to the settlement of individual claims (e.g., lawyer's fees, claims investigation expenses). The summary statistics, including the standard deviation $(\mathrm{SD})$, the first quartile $\left(Q_{1}\right)$ and the third quartile $\left(Q_{3}\right)$ are reported in Table 2.

To visualize the relationship, scatter plots in Fig. 4 are constructed on the real dollar scale and on the log scale. There seems to be an upper tail dependence in the data.

Using the notation in (1), the log-likelihood function for the data $\left\{\left(x_{i}, y_{i}\right)\right\}_{i=1}^{n}$ is given by $\boldsymbol{L}\left(\theta, a, b, \alpha_{1}, \alpha_{2} ; x, y\right)=\sum_{i=1}^{n} \log \left[c\left(F\left(x_{i} ; \alpha_{1}\right), G\left(y_{i} ; \alpha_{2}\right)\right)\right]+\sum_{i=1}^{n}\left[\log f\left(x_{i} ; \alpha_{1}\right)+\log g\left(y_{i} ; \alpha_{2}\right)\right]$,

where $\alpha_{1}$ and $\alpha_{2}$ are the parameters in the marginal distributions, and $\theta, a$ and $b$ are parameters in the copula function. Rather than a full maximum likelihood estimation, one of the more attractive estimation methods is the two-stage maximum likelihood estimation, also know as inference function for margins (IFM); see Joe (1997). The IFM first obtains estimates, $\widehat{\alpha}_{1}$ and $\widehat{\alpha}_{2}$, of parameters in the marginals by maximizing $\sum_{i=1}^{n} \log f\left(x_{i} ; \alpha_{1}\right)$ and $\sum_{i=1}^{n} \log g\left(y_{i} ; \alpha_{2}\right)$, and then computes estimates of the parameters $\theta, a$, and $b$ by maximizing

$$
\sum_{i=1}^{n} \log \left[c\left(F\left(x_{i} ; \widehat{\alpha}_{1}\right), G\left(y_{i} ; \widehat{\alpha}_{2}\right)\right)\right] .
$$

To determine the appropriate marginals for the ALAE and loss variables, (Frees and Valdez 1998) and (Frees 2018) overlaid the fitted Pareto cdf and the empirical cdf and found the two curves are reasonably close to each other for both variables. Since we ignore the mild censoring in the loss variable, we reassess the fit using P-P plots. The P-P plots in Fig. 5 indicate that the Pareto margins fit the data well. Therefore, we model both of the marginals using the Pareto distribution with the cdf of the from $1-\left[1+\left(x / \alpha_{i 1}\right)\right]^{-\alpha_{i 2}}, i=1,2$, where $\alpha_{i 1}$ is the scale parameter and $\alpha_{i 2}$ is the shape parameter. From the loss marginal, $\widehat{\alpha}_{1}=\left(\widehat{\alpha}_{11}, \widehat{\alpha}_{12}\right)=(16228.15,1.238)$, and for ALAE, $\widehat{\alpha_{2}}=\left(\widehat{\alpha}_{21}, \widehat{\alpha}_{22}\right)=(15133.6,2.223)$. Plugging in $\widehat{\alpha}_{1}$ and $\widehat{\alpha}_{2}$ in (28), we then ascertain estimates in Table 3 by maximizing (28). Genest et al. (1998) ignored the censoring in the loss data, so did we in this paper. The pseudo log-likelihood estimation maximizes (28) using nonparametric, empirical distribution estimates of $F$ and $G$. The results are of little differences and therefore not reported here.

Based on the scatter plots, we select survival Clayton (SClayton) (a 180 degrees rotation of the Clatyon copula) and Gumbel copulas, in addition to Frank and Gaussian copulas. The two-parameter survival BB1 (SBB1) is selected by the SelectCopula() in R Vine package as the best-fit bivariate copula model. The Roptim() function is employed to compute the IFM estimates.

In addition to standard errors (SE) of the estimators, we also report the resulting loglikelihoods (IFML) in (28) and AIC for comparison purposes. The parameter $\theta$ (and $\delta$ for the survival BB1 copula) is the one originated from the initial copula, $a$ and $b$ are parameters injected by the UW distortion. The estimate of the correlation parameter $\rho$ in

Table 2 Summary statistics of Losses-ALEA data

\begin{tabular}{lllllllll}
\hline & $\boldsymbol{n}$ & Mean & Median & SD & Min & Max & $\boldsymbol{Q}_{\mathbf{1}}$ & $\boldsymbol{Q}_{\mathbf{3}}$ \\
\hline ALAE & 1500 & 12588 & 5471 & 28145.64 & 15 & 501863 & 2333 & 12572 \\
LOSS & 1500 & 41208 & 12000 & 102747.7 & 10 & 2173595 & 4000 & 35000 \\
\hline
\end{tabular}



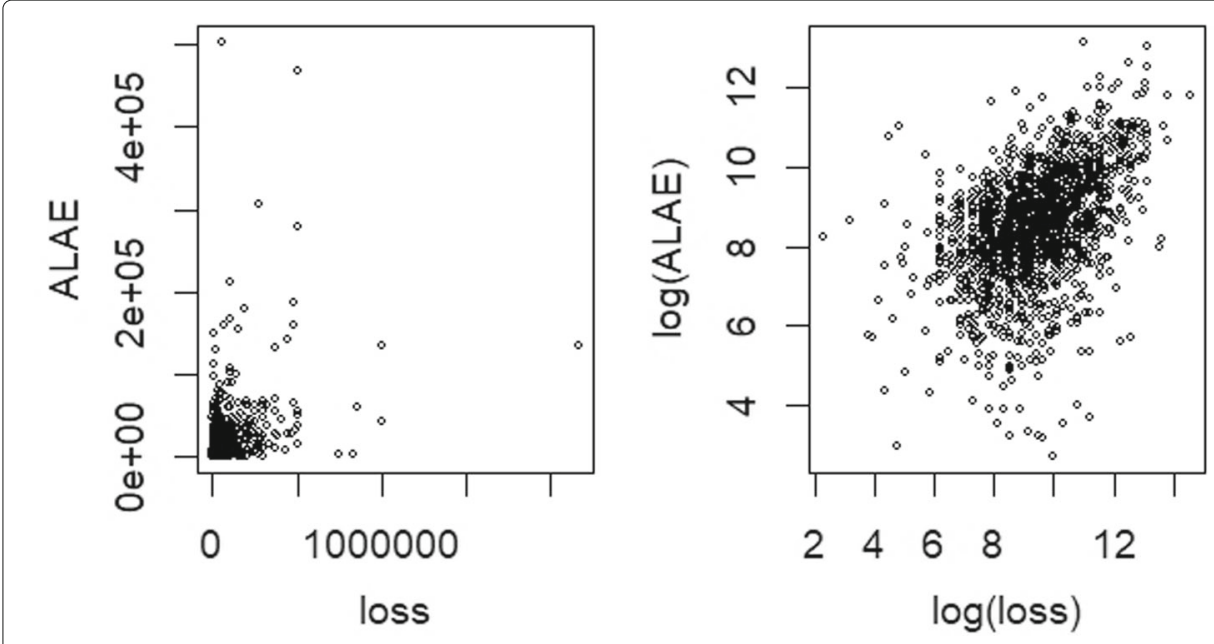

Fig. 4 Scatter plots of ALAE versus Loss

th Gaussian copula is denoted by $\widehat{\theta}$ in Table 3. The estimate of $\theta$ resulting from fitting the initial copula is used as a starting point in the optim() for fitting UW-distorted models and the Beta(B)-distorted copulas (Samanthi and Sepanski 2019). The dash symbol (-) is used for models with a lower or an upper tail dependence coefficient of 0 . Note that the sample Kendall's tau is 0.315 .

The Cramer-von Mises goodness-of-fit test statistic (CvM) for copulas in Genest et al. (2009) measures the sum of square deviations between the empirical cdf and an estimated copula cdf. Larger CvM values are less desirable. The bootstrap approach detailed in Genest et al. (2009) was used to calculate $p$-values. Note the sample size is 1500 and we used 1000 bootstrap replications. The results, e.g., the CvM for the UW-SBB1 copula is 0.296 with a $p$-value of 0.127 , are tabulated in Table 4. Apart from the Frank, Gaussian, and SClayton copulas, the copulas listed in Table 4 provide an adequate fit to the data.
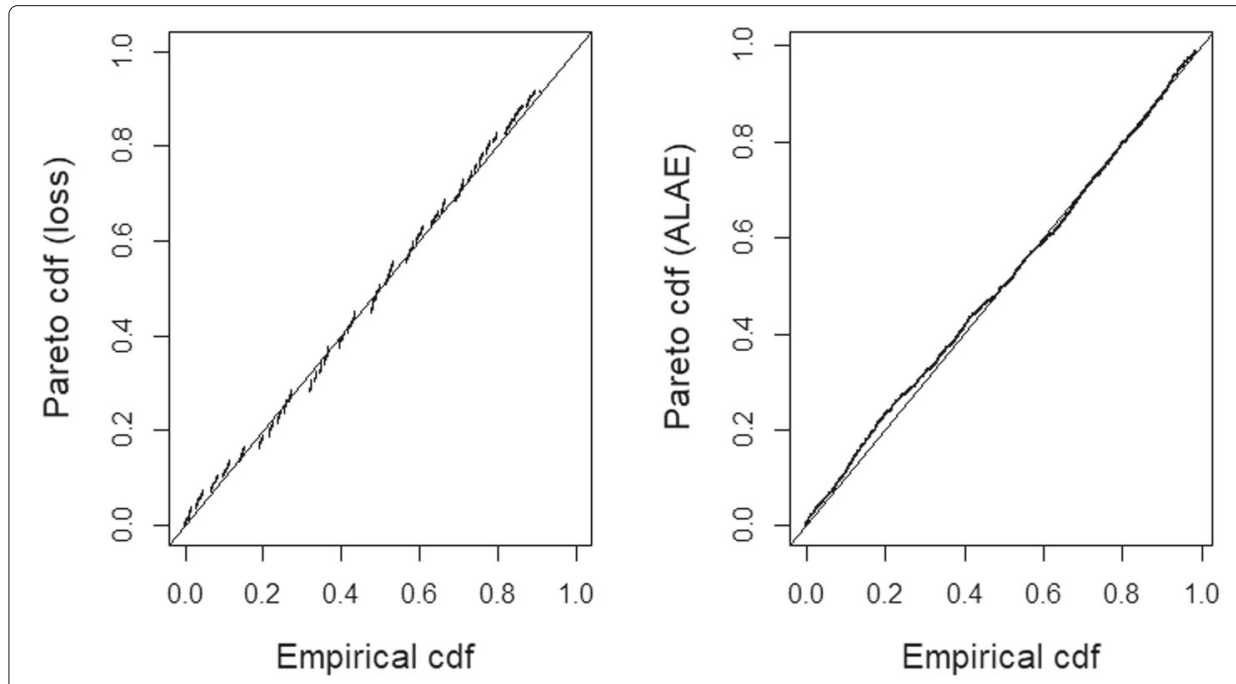

Fig. 5 P-P plots for ALAE and Loss 
Table 3 IFML, AIC, $\widehat{\tau}_{,} \widehat{\lambda}_{L}, \widehat{\lambda}_{U}$, and parameter estimates with standard errors in parentheses for the base, UW and Beta distorted copula models

\begin{tabular}{lllllllll}
\hline Family & IFML & AIC & $\widehat{\boldsymbol{\tau}}$ & $\widehat{\boldsymbol{\lambda}}_{\boldsymbol{L}}$ & $\widehat{\boldsymbol{\lambda}}_{\boldsymbol{U}}$ & $\widehat{\boldsymbol{\theta}}$ & $\widehat{\boldsymbol{a}}$ & $\widehat{\boldsymbol{b}}$ \\
\hline Frank & 172.6 & -343.1 & 0.316 & - & - & $3.11(0.17)$ & & \\
Gaussian & 183.8 & -365.7 & 0.319 & - & - & $0.48(0.02)$ & & \\
SClayton & 198.9 & -395.8 & 0.278 & - & 0.41 & $0.77(0.05)$ & & \\
Gumbel & 204.9 & -407.8 & 0.306 & - & 0.38 & $1.44(0.03)$ & & \\
SBB1 & 206.2 & -408.4 & 0.307 & 0.13 & 0.35 & $0.60(0.06)$ & & \\
SBB1 & & & & & & $1.11(0.03)$ & & \\
UW-SClayton & 204.2 & -402.5 & 0.269 & - & 0.36 & $0.53(0.10)$ & $0.90(0.03)$ & $0.98(0.06)$ \\
UW-Frank & 205.5 & -405 & 0.311 & - & 0.35 & $0.38(0.36)$ & $0.72(0.03)$ & $0.63(0.46)$ \\
UW-Gaussian & 205.1 & -404.2 & 0.319 & - & 0.36 & $0.02(0.04)$ & $0.71(0.03)$ & $0.14(0.36)$ \\
UW-SBB1 & 207.8 & -407.6 & 0.317 & 1 & 0.32 & $0.24(0.15)$ & $0.78(0.07)$ & $0.97(0.30)$ \\
UW-SBB1 & - & - & - & - & - & $1.01(0.02)$ & & \\
B-SClayton & 204.7 & -403.3 & 0.287 & - & 0.53 & $0.89(0.72)$ & $1.24(0.74)$ & $0.89(0.33)$ \\
B-Frank & 206.7 & -407.3 & 0.314 & - & 0.40 & $1.35(0.25)$ & $1.05(0.16)$ & $0.68(0.10)$ \\
B-Gaussian & 204.3 & -402.6 & 0.216 & - & 0.15 & $0.26(0.05)$ & $1.10(0.67)$ & $0.89(0.13)$ \\
B-SBB1 & 206.6 & -405.3 & 0.312 & 0.08 & 0.35 & $0.47(0.54)$ & $1.34(0.63)$ & $0.92(0.37)$ \\
B-SBB1 & & & & - & - & $1.13(0.05)$ & & \\
\hline
\end{tabular}

The distortion induced copulas, as expected, outperform the initial copulas in terms of log-likelihoods. There is a sizable improvement in the log-likelihoods of the UW-Frank, UW-Gaussian, Beta-Frank, and Beta-Gaussian copulas over the Frank and Gaussian copulas. It may due to the fact that the distorted copulas can accommodate the upper tail dependence in the data. As indicated in Table 4, distortions can improve the goodnessof-fit in terms of the CvM statistic. While more parameters are expected to yield better log-likelihood results, the AIC that penalized for having more parameters indicates that the two-parameter survival BB1 is the winner among the chosen copulas for fitting this particular data set. However, the estimated Kendall's tau calculated based on the estimated survival BB1 model seems to deviate from the sample Kendall's tau more than the ones based on the estimated UW-SBB1 and B-SBB1 models. The upper tail coefficient estimates from models with better performance in terms of IFML seem to suggest the upper tail dependence exists in the data. The standard errors of estimates from the UW-distorted copulas are smaller than those from Beta distorted copulas, although they perform comparably in terms of the AIC and log-likelihood.

\section{Concluding remarks}

This paper constructs a new family of copulas by employing the unit Weibull distributional distortion function. With an additional two parameters in the unit Weibull distortion, the new family of copulas allows for more modeling flexibility and versatility. Note also that the initial copula is a special case of the UW-distorted copulas and

Table 4 Cramer-von Mises statistics and p-values in parentheses for the base, UW and Beta distorted copula models

\begin{tabular}{llllll}
\hline Model & Frank & Gaussian & SClayton & Gumbel & SBB1 \\
\hline Base & $0.384(0.039)$ & $0.381(0.043)$ & $0.398(0.033)$ & $0.308(0.077)$ & $0.316(0.076)$ \\
UW-Distorted & $0.297(0.075)$ & $0.309(0.086)$ & $0.354(0.072)$ & & $0.296(0.127)$ \\
B-Distorted & $0.297(0.094)$ & $0.339(0.061)$ & $0.302(0.110)$ & & $0.305(0.075)$ \\
\hline
\end{tabular}


therefore the proposed family of copulas preserves properties in the initial copula. The UW-distortion remains Archimedean if the initial copula is Archimedean. Intuitively, and as seen in the empirical results, the proposed UW-distorted copula outperforms its initial copula. The family of UW-Clayton copulas, for instance, contains the following copulas as special cases: Clayton, Fréhet upper bound, Gumbel, and BB3 copulas. The unit Weibull distortion can transform an existing copula without upper tail dependence, e.g., the Clayton and Frank copulas, into one with upper tail dependence.

The transformation mechanism in (6) that results in the unit Weibull distortion can be applied to other random variables with different cumulative distribution functions. For example, instead of the Weibull cdf, a Burr or Gompertz or Log-logistic distribution may be employed in (6), which yields he following possible distortions: (i) Unit-Burr: $T(u)=$ $\left[1+(-\log (u))^{b}\right]^{-a}$; (ii) Unit-Gompertz: $T(u)=\exp \left[-a\left(b^{-\log (u)}-1\right) / \log b\right]$; and (iii) Unit-Log Logistic: $T(u)=\left[1+b(-\log (u))^{a}\right]^{-1}$. The admissibility of the above twoparameter distortions will be further investigated and distortions of multivariate copulas of dimension more than two may also be of interest. Moreover, naturally, the next step is to investigate distortions of multivariate copula distributions. Unlike distortions of bivariate copulas, distortions of multivariate copulas require more care and are being explored for future publications.

\section{Abbreviations}

UW: Unit Weibull; ALAE: Allocated loss adjustment expenses; IFM: Inference function for margins; IFML: Inference function for margins log-likelihood; AIC: Akaike information criterion; SD: Standard deviation; SE: Standard errors

\section{Acknowledgements}

The authors are grateful to the reviewers for their careful reading and suggestions that helped improve the paper.

\section{Authors' contributions}

The authors carried out this work and drafted the manuscript collaboratively. All author(s) read and approved the final manuscript.

Funding

Not applicable.

Availability of data and materials

The data set generated and/or analyzed during the current study is available in the R copula package.

\section{Competing interests}

The authors declare that they have no competing interests.

Received: 6 May 2020 Accepted: 15 September 2020

Published online: 06 October 2020

\section{References}

Balakrishnan, N., Lai, C. D.: Continuous bivariate distributions. Springer, New York (2009)

Bingham, N. H., Goldie, C. M., Teugels, J. L.: Regular variation. Cambridge University Press, Cambridge (1989)

Buldygin, V. V., Klesov, O. I., Steinebach, J. G.: PRV property and the $\varphi$-asymptotic behavior of solutions of stochastic differential equations. Lith. Math. J. 47(4), 361-378 (2006)

Cooray, K.: A new extension of the FGM copula for negative association. Commun. Stat. Theory Methods. 48(8), 1902-1919 (2019)

Di Bernardino, E., Rulliere, D.: On certain transformations of Archimedean copulas: Application to the non-parametric estimation of their generators. Depend. Model. 1, 1-36 (2013)

Durante, F., Foschi, R., Sarkoci, P.: Distorted copulas: constructions and tail dependence. Commun. Stat. Theory Methods. 39(12), 2288-2301 (2010)

Frank, M. J.: On the simultaneous associativity of $F(x, y)$ and $x y-F(x, y)$. Aequationes Math. 19(1), 194-226 (1979)

Frees, E: Loss data analytics. arXiv preprint arXiv:1808.06718, 1-319 (2018)

Frees, E. W., Valdez, E. A.: Understanding relationships using copulas. N. Am. Actuar. J. 2(1), 1-25 (1998)

Genest, C., Ghoudi, K., Rivest, L. P.: Understanding relationships using copulas by Edward Frees and Emiliano Valdez, January 1998. N. Am. Actuar. J. 2(3), 143-149 (1998)

Genest, C., MacKay, J.: The joy of copulas: bivariate distributions with uniform marginals. Am. Stat. 40(4), 280-283 (1986) 
Genest, C., Remillard, B., Beaudoin, D.: Goodness-of-fit tests for copulas: A review and a power study. Insur. Math. Econ. 44(2), 199-213 (2009)

Gudendorf, G., Segers, J.: Extreme-value copulas. In: Copula Theory and Its Applications Lecture Notes in Statistics, pp. 127-145. Springer, Berlin, Heidelberg, (2010)

Hua, L, Joe, H.: Intermediate tail dependence: a review and some new results. In: Stochastic Orders in Reliability and Risk Lecture Notes in Statistics, pp. 291-311. Springer, New York, NY, (2013)

Joe, H.: Multivariate Models and Dependence Concepts. Chapman \& Hall, London (1997)

Joe, H.: Dependence modeling with copulas. CRC Press, Boca Raton, FL (2015)

Lee, M.-L. T.: Properties and applications of the Sarmanov family of bivariate distributions. Commun. Stat. Theory Methods. 25(6), 1207-1222 (1996)

Morgenstern, D.: Einfache beispiele zweidimensionaler verteilungen. Mitteilingsblatt fur Math. Stat. 8, 234-235 (1956)

Morillas, P. M.: A method to obtain new copulas from a given one. Metrika. 61(2), 169-184 (2005)

Nazemi, A., Elshorbagy, A.: Application of copula modelling to the performance assessment of reconstructed watersheds. Stoch. Environ. Res. Risk Assess. 26(2), 189-205 (2012)

Nelsen, R. B.: An introduction to copulas. Springer, New York (2006)

Samanthi, R. G. M., Sepanski, J.: A bivariate extension of the beta generated distribution derived from copulas. Commun. Stat. Theory Methods. 48(5), 1043-1059 (2019)

Schweizer, B., Sklar, A.: Probabilistic metric spaces. Elsevier North, Holland (1983)

Sharifonnasabi, Z., Alamatsaz, M. H., Kazemi, I.: A large class of new bivariate copulas and their properties. Braz. J. Probab. Stat. 32(3), 497-524 (2018)

Sklar, M.: Fonctions de repartition a n dimensions et leurs marges. Publ. Inst. Stat. de I'Universite Paris. 8, 229-231 (1959) Valdez, E. A., Xiao, Y:: On the distortion of a copula and its margins. Scand. Actuar. J. 4, 292-317 (2011)

Xie, J., Yang, J., Zhu, W.: A family of transformed copulas with a singular component. Fuzzy Sets Syst. 354, $20-47$ (2019)

\section{Publisher's Note}

Springer Nature remains neutral with regard to jurisdictional claims in published maps and institutional affiliations.

\section{Submit your manuscript to a SpringerOpen ${ }^{\circ}$ journal and benefit from:}

- Convenient online submission

- Rigorous peer review

Open access: articles freely available online

- High visibility within the field

- Retaining the copyright to your article

Submit your next manuscript at $\gg$ springeropen.com 